\title{
The canonical FGF-FGFR signaling system at the molecular level
}

Patrycja Szybowska ${ }^{1,3}$, Ellen Margrethe Haugsten ${ }^{1,3}$, Antoni Wiedlocha*2,3,4

${ }^{1}$ Department of Tumor Biology Institute for Cancer Research, The Norwegian Radium Hospital, Oslo University Hospital, Montebello, Norway ${ }^{2}$ Department of Molecular Cell Biology, Institute for Cancer Research, The Norwegian Radium Hospital, Oslo University Hospital, Montebello, Norway

${ }^{3}$ Center for Cancer Cell Reprogramming, Institute of Clinical Medicine, Faculty of Medicine, University of Oslo, Montebello, Norway

${ }^{4}$ Military Institute of Hygiene and Epidemiology, Warsaw, Poland

Abstract

Extracellular signaling molecules, among them the fibroblast growth factors (FGFs), enable cells to communicate with neighboring cells. Such signaling molecules that receive and transmit a signal require specific tyrosine kinase receptors located at the cell surface (fibroblast growth factor receptors, FGFRs). The binding of a signaling molecule to its specific receptor results in receptor dimerization and conformational changes in the cytoplasmic part of the receptor. The conformational changes lead to trans-autophosphorylation of the tyrosine kinase domains of the receptors and subsequently to induction of several downstream signaling pathways and expression of appropriate genes. The signaling pathways activated by FGFs control and coordinate cell behaviors such as cell division, migration, differentiation, and cell death. FGFs and their transmembrane receptors are widely distributed in different tissues and participate in fundamental processes during embryonic, fetal, and adult human life. The human FGF/FGFR family comprises 22 ligands and 4 high affinity receptors. In addition, FGFs bind to low affinity receptors, heparan sulfate proteoglycans at the cell surface. The availability of appropriate ligand/receptor pair, combined with the co-receptor, initiates signaling. Inappropriate FGF/FGFR signaling can cause skeletal disorders, primarily dwarfism, craniofacial malformation syndromes, mood disorders, metabolic disorders, and Kallman syndrome. In addition, aberrations in FGF/FGFR signaling have already been reported in several types of malignant diseases. Knowledge about the molecular mechanisms of FGF/FGFR activation and signaling is necessary to understand the basis of these diseases.

Keywords

Fibroblast growth factors • fibroblast growth factor receptors • cellular signaling • tissue homeostasis

Received: 16.10.2020, Accepted: 24.02.2021

\section{Introduction}

The sophisticated organization of tissues in multicellular organisms requires communication between cells. They do it by producing regulatory proteins, so-called growth factors that are sent out to the cell exterior to instruct neighboring cells in a tissue. The receiver cells present specific tyrosine kinase receptors at their cell surface that recognize specific ligands. The fibroblast growth factors (FGFs) and FGFreceptors (FGFRs) constitute such a communication system.

FGFs and FGFRs have evolved by gene amplification and differentiation as a regulatory signaling system that is operative in invertebrates as well as in vertebrates [1, 2]. Their phylogenesis goes back to the time and events called the Cambrian Explosion, half a billion years ago [3, 4]. The long evolution of this growth factor/receptor system implies that FGF-induced signaling controls fundamental processes during embryonic, fetal, and adult life $[5,6,7,8]$. The FGF-FGFR axis of signaling is a key regulator of mesenchymal-epithelial communication and is thus required for organogenesis. The FGFFGFR signaling also plays crucial roles in tissue homeostasis [9]. They represent fundamental mediators of such cell-to-cell communication. Therefore, imbalances in FGF-FGFR signaling cause a wide spectrum of pathological conditions, including different types of developmental and metabolic disorders as well as malignant diseases [10].

The pleiotropic biological action of FGFs is exerted through binding to and activation of four high affinity, tyrosine kinase, cell surface FGF receptors (FGFR1-4) and splicing variants for three of them (FGFR1-3). The active receptor further initiates various intracellular, downstream signaling pathways leading to positive and negative regulation of cell differentiation, proliferation, migration, and survival. The different biological responses 
induced by FGF-FGFR signaling seem to depend on the type of receiver cell.

\section{Ligands for Fibroblast Growth Factor Receptors}

The FGFRs are mainly activated by FGFs. In humans, the FGF family comprises 22 strucuturally related polypeptides (FGF1-23) [11]. Since human FGF15 and mouse FGF19 have not been identified and they have $15 \%$ amino acid identity, they have been suggested to be orthologs [12]. All FGFs are recognized by a highly conserved core of around 140 amino acids [13]. They exhibit a beta-trefoil structure [14, 15]. The beta-trefoil protein fold belongs to the evolutionary oldest protein folds and contains 12 beta-strands, which form six two-stranded beta-hairpins [16]. The FGF protein family represents one of the largest signaling families in vertebrates.

The mammalian FGFs can be divided into three subgroups based on their way of action: the paracrine (canonical) FGFs, the endocrine FGFs, and the intracrine (intracellular) FGFs (Fig. 1).

The paracrine (canonical) FGFs can additionally be divided into 5 subfamilies based on their evolutionary relationship: FGF1 subfamily (FGF1, FGF2), FGF4 subfamily

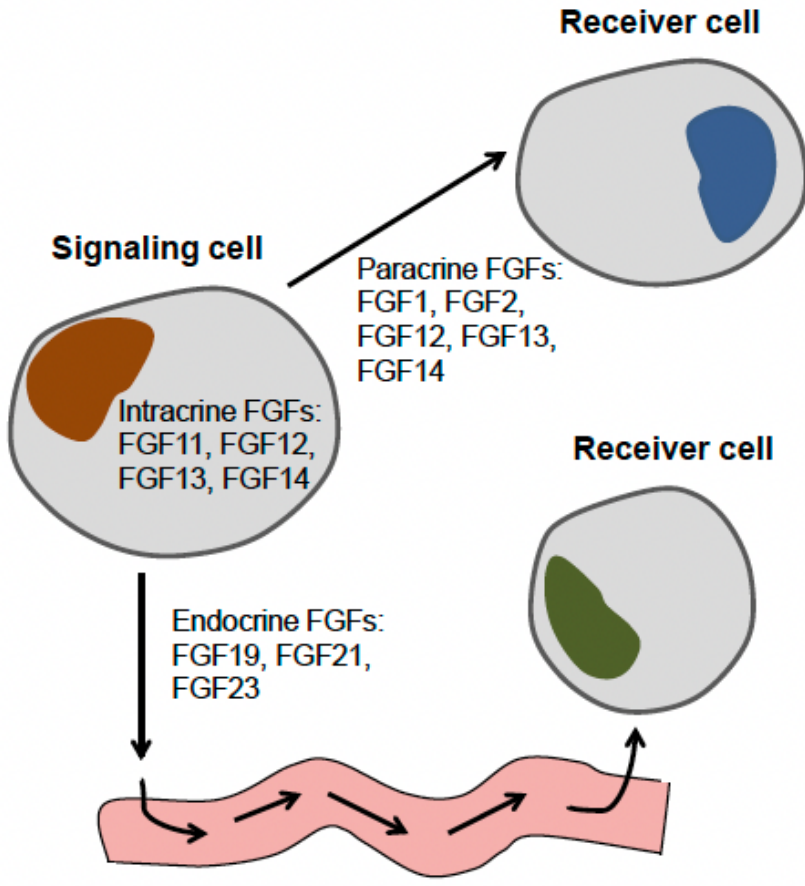

Figure 1. The FGF family. The mammalian FGFs can be divided into three subgroups based on their way of action. The intracrine FGFs are not secreted and remain inside the signaling cell. The paracrine and endocrine FGFs are secreted and bind to receiver cells but the endocrine FGFs mainly play roles in an endocrine manner via the blood circulation system.
(FGF4, FGF5, FGF6), FGF7 subfamily (FGF3, FGF7, FGF10, FGF22), FGF8 subfamily (FGF8, FGF17, FGF18) and FGF9 subfamily (FGF9, FGF16, FGF20) [1]. FGF1 and FGF2 were the first FGFs discovered and their original names - acidic and basic FGFs (aFGF and bFGF) - are used in earlier publications [17]. FGF1 is the only FGF that activates all FGFRs and their splicing variants similarly [11]. Both FGF1 and FGF2 lack the classical secretory signal peptides and are released through a nonclassical ER-Golgi independent exocytic pathway as well as from damaged cells $[18,19]$. The canonical FGFs also have relatively high affinity to heparan sulfate (HS) and heparin (Hp) [20]. Upon secretion they are trapped by binding to $\mathrm{HS}$ in the extracellular matrix and thus usually act in a paracrine and/or autocrine manner. Moreover, binding of paracrine FGFs to HS stabilizes the structure of the ligand as well as the receptor-ligand complex and enhances resistance to proteolysis [21, 22, 23].

FGF15/19, FGF21, and FGF23 have evolved as the endocrine FGF subgroup, and are specific to vertebrates [24]. These FGFs have lost their HS/Hp binding affinity. Therefore, the endocrine FGFs are not retained in the location where they are produced and can act as secreted hormones. Instead of $\mathrm{HS}$, they require the presence of Klotho proteins (aKlotho and $\beta$ Klotho) or Klotho-related proteins (KLPH) that act as coreceptors to form the active complex with FGFR. Endocrine FGF knockout mice have proved the important roles of these FGFs in regulating metabolism $[25,26]$.

The intracrine (intracellular) subgroup includes FGF11, FGF12, FGF13, and FGF14. Since they have a genomic structure and protein fold similar to the other FGFs, but lack the ability to bind and activate FGFRs, they have been called the homologue FGFs $[17,27,28]$. The intracellular FGFs lack signal sequence, so they are not secreted and remain inside the cell, mainly in the nucleus, and are able to interact with a separate set of target proteins than canonical and endocrine FGFs [12]. The biological function of the intracellular FGFs is not very well known, but some have been implicated in the regulation of voltage-gated sodium channels [29, 30]. The intracellular FGFs also interact with other intracellular proteins, such as, for example, FGF13, which interacts with microtubules [31].

It should be mentioned that although FGF1, FGF2, and FGF3 act in a paracrine or autocrine fashion, they have also been found in the nucleus as endogenous as well as exogenous proteins [32, 33, 34, 35, 36]. The nuclear function of FGF1 and 2 is not fully understood but nuclear FGF1 might play a role in regulation of the cell cycle, cell differentiation, survival, and apoptosis [37]. 


\section{Fibroblast growth factor receptors}

In humans, there are four high affinity FGFRs. They belong to the receptor tyrosine kinase family. They are singlepass membrane receptors localized at the cell surface and are comprised of an extracellular, a transmembrane, and a cytosolic domain [38, 39].

In addition to the four high affinity FGF receptors at the cell surface, the paracrine FGFs also bind to heparan sulfate proteoglycans (HSPGs) that act as low affinity receptors at the cell surface. HSPGs exist as transmembrane or lipidanchored proteins at the cell surface or as diffusible proteins in the extracellular environment. HSPGs are composed of a core protein covalently attached to one or more heparan sulfate chains. The heparan sulfate chains are linear polysaccharides of variably sulfated repeating disaccharide units [40]. HS/Hp act as powerful cofactors/receptors, as they are required for most secreted FGFs for signaling (see text below) [20, 41]. Even though FGFs have around 100 -fold lower affinity for HSPGs than for FGFRs, HSPGs play important roles in FGF signaling, as co-receptors but also as storage reservoirs for the growth factors [21].

\section{FGFR activation}

FGFRs consist of two or three extracellular immunoglobulin (Ig)-like domains (Ig-like domain I-III), a transmembrane domain and an intracellular split tyrosine kinase domain (Fig. 2a). The Ig-like I domain and the serine-rich sequence located in the linker between Ig-like I and II domains, also called the acid box, play a role in autoinhibition of the receptor [38]. Ig-like II domain contains a positively charged HSPG binding site. Ig-like II and III domains and the region between these domains are forming the binding sites for FGFs [42]. FGFs differ in their FGFR binding affinities. FGFR1-3 are alternatively spliced in Ig-like domain III to generate FGFRIIIb or FGFRIIIc variants that determines FGF binding specificity [9]. FGFRIIlb splice variants are more likely to be expressed in epithelial tissue while FGFRIIlc splice variants are expressed in the mesenchymal tissue [9]. On the other hand, the ligands for FGFRIIlb isoforms are often secreted from mesenchymal cells whereas the ligands for FGFRIIlc isoforms are often secreted from epithelial cells. In this way FGFs and FGFRs enable an epithelial-mesenchymal crosstalk [43].

The FGF-FGFR interaction triggers receptor dimerization and activation of the tyrosine kinase domain of the receptors (Fig. 2b). The molecular mechanism that leads to FGFR activation is still somewhat unclear but several models have been proposed. Two ternary complex models of FGF, FGFR, and $\mathrm{HS} / \mathrm{Hp}$, the asymmetric and the symmetric model, are now recognized as the most probable. Both models are based on crystallographic studies. In the asymmetric model, FGF:FGFR:Hp are thought to associate in a 2:2:1 complex. $\mathrm{Hp}$ facilitates FGFs dimerization bringing together the two FGFRs. In addition, $\mathrm{HS} / \mathrm{Hp}$ binds to Ig-like domain II of only one of the FGFRs in the complex [44]. In the symmetric model, a FGF:FGFR:Hp 1:1:1 ternary complex recruits a second similar 1:1:1 complex via receptor dimerization forming a dimeric 2:2:2 complex. FGF has two receptor-binding sites (a primary and a secondary) for interaction with both FGFRs in the dimer. Each receptor binds to both ligands through the complementary primary and secondary ligand-binding sites located within Ig-like domains II and III and the linker between them. In addition, the two receptors interact with each other directly [45]. The role of $\mathrm{HS} / \mathrm{Hp}$ in FGF/FGFR assembly varies between the two models. In the asymmetric model HS/ $\mathrm{Hp}$ bridges the ligand-receptor complex by mediating growth factor dimerization while binding one of the two receptors. In the symmetric model, $\mathrm{HS} / \mathrm{Hp}$ increases stability of the ligandreceptor complex and thus helps the dimerization but is not required for dimerization [46].

At present, most studies support the symmetric model of assembly, since the symmetric model fits with the finding that receptors can dimerize in the absence of ligand [47, 48]. The asymmetric model is still not excluded, and it has been proposed that both models might coexist [49]. The two models
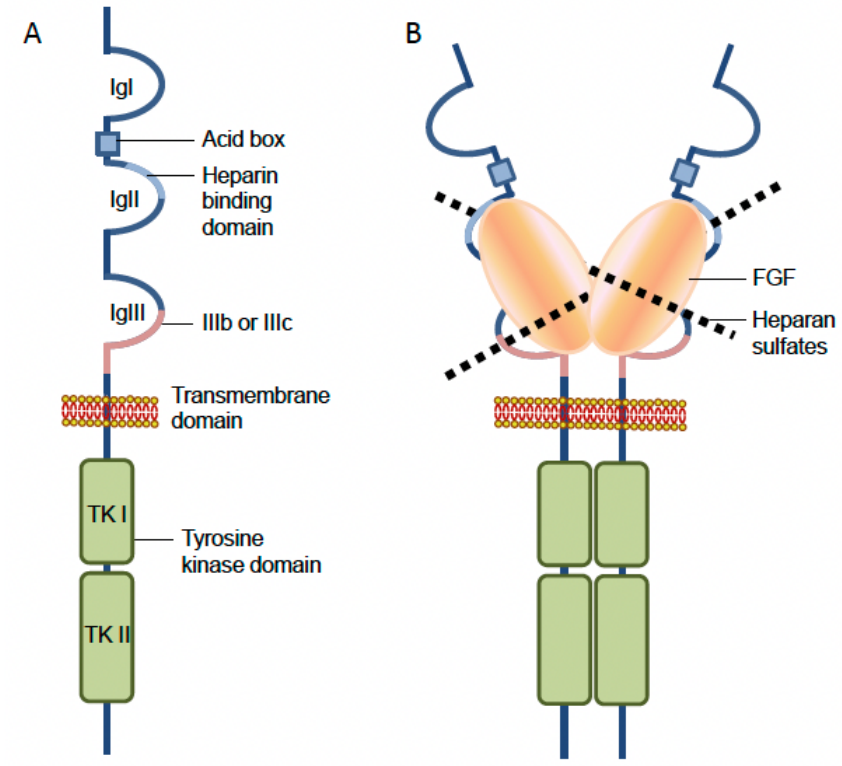

Figure 2. A schematic presentation of the structure and the activation of FGFRs. (A) FGFRs consist of two or three extracellular immunoglobulin (Ig)-like domains (Igl-IgllI), a transmembrane domain and an intracellular split tyrosine kinase domain (TKI and TKII). An acid box is located between Igl and Igll. The Igll contains also a heparin binding domain. IgIII in FGFR1-3 is alternatively spliced to Iglllb or Iglllc variants. (B) Activation of FGFRs. FGF-FGFR interaction in the presence of heparan sulfate triggers receptor dimerization and activation of the tyrosine kinase domain of the receptors. 
are based on crystallographic studies of different ligands/ receptors. It is possible that differences in specificity of ligand binding motifs, distinct $\mathrm{HS} / \mathrm{Hp}$-binding sites for FGFs and FGFRs, and structural changes in FGFRs after ligand binding might explain the discrepancies between the two models [50].

Activation of FGFR signaling by the paracrine FGFs is regulated by the pattern of sulfation and length of the HS chains. Generally, the higher level of sulfation of HS chains and the longer chains facilitate the formation of the FGF:FGFR:HS/Hp complex. Shorter HS chains and lower sulfation levels have the same capability but with less efficiency [51].

Ligand binding and receptor dimerization lead to the activation of the tyrosine kinase domain in the intracellular part of the receptor. Each kinase domain phosphorylates tyrosine residues present in the intracelullar part of the other receptor [48]. This process is called trans-autophosphorylation [52]. FGFR1 has seven tyrosine residues that are phosphorylated in a specific order (Y463, Y583, Y485, Y653, Y654, Y730, and $Y 766)[53,54]$. At the end of this strictly ordered transautophosphorylation cascade, the tyrosine kinase activity of the receptor is increased up to 1,000 -fold [55].

\section{Canonical FGFR signaling}

The four classical, major signaling pathways activated by FGF/FGFRs include the RAS-MAPK (mitogen-activated protein kinase) pathway, the PI3K (phosphatidylinositol4,5-bisphosphate 3-kinase)-AKT pathway, the PLCY (phospholipase Cy)/PKC (protein kinase C) pathway, and the STAT (signal transducer and activator of transcription) pathway [11]. Direct recruitment of signaling proteins or adaptor proteins to the phosphorylated tyrosines in the kinase domain of the receptor mediates FGFR signaling. Proteins bind directly to the phosphorylated tyrosines via an $\mathrm{SH} 2$ ( $\mathrm{Src}$ homology 2) domain or a PTB (phosphotyrosine binding) domain. Activated FGFR phosphorylates bound proteins, resulting in recruitment of additional signaling molecules. FGFR substrate $2 \alpha$ (FRS2 $\alpha$ ) is constitutively bound to FGFRs and is phosphorylated on tyrosine residues by the activated FGFR kinase leading to activation of RAS-MAPK and PI3KAKT signaling pathways [11]. The phosphorylated tyrosines in FRS2 serve as docking sites, recruiting the $\mathrm{SH} 2$ domaincontaining adaptors GRB2 (growth factor receptor-bound 2) and SHP2 (Src homology region 2 domain-containing phosphatase-2, also known as PTPN11; tyrosine-protein phosphatase non-receptor type 11) [56, 57]. This again leads to recruitment of the guanine nucleotide exchange factor, SOS (son of sevenless homology) [58]. SOS exchanges GDP for GTP in RAS, resulting in activation of RAF and distinct MAPKs including ERK1/2, p38, and JNK (c-Jun N-terminal kinase) [11]. Activation of the RAS-MAPK pathway leads to phosphorylation and activation of E26 transformation-specific (ETS) transcription factors that regulate expression of target genes [11].

PI3K-AKT activation requires recruitment of the docking protein GAB1 (GRB2-associated-binding protein1) by GRB2 to the signaling complex at the plasma membrane [59]. PI3K then phosphorylates PIP2 (phosphatidylinositol-4,5-bisphosphate) generating PIP3 (phosphatidylinositol-3,4,5-trisphosphate) in the plasma membrane. This leads to translocation of PDK1 (phosphoinositide-dependent kinase 1) and AKT (also known as protein kinase $B(P K B)$ ) to the plasma membrane. Both PDK1 and AKT bind directly to PIP3 via their pleckstrin homology $(\mathrm{PH})$ domains. AKT is then phosphorylated and activated by PDK1. The PI3K-AKT pathway activates and inhibits many target molecules. For example, AKT phosphorylates TSC2 (tuberous sclerosis complex 2) leading to its inactivation. This again leads to activation of the mTOR (mammalian target of rapamycin) complex [11, 60]. AKT also phosphorylates pro-apoptotic proteins such as BAD (Bcl-2 antagonist of cell death) and inhibits their function [61]. Thus AKT activation is often connected with cell survival.

To activate the PLCY/PKC pathway, PLCY has to bind directly, via its $\mathrm{SH} 2$ domain, to the phosphorylated $\mathrm{Y} 766$ in the FGFR C-terminal part. Once bound, PLCY is activated by a phosphorylation event mediated by the FGFR tyrosine kinase [54]. Activated PLCY catalyzes the hydrolysis of PIP2 to produce inositol triphosphate (IP3) and diacylglycerol (DAG). IP3 production leads to an increase in intracellular $\mathrm{Ca}^{2+}$ resulting in activation of $\mathrm{Ca}^{2+} /$ calmodulin-dependent kinase II (CaMKII). DAG recruits and activates PKC. Adaptor protein GRB14 also binds to phosphorylated $\mathrm{Y} 766$ and inhibits activation of PLCY [11].

Transcription factors in the STAT family such as STAT1, STAT3, and STAT5 can also be phosphorylated by active FGFRs [11]. STAT3 binds directly to the phosphorylated Y677 of FGFR1 and is thought to be activated upon overexpression of FGFR1 or FGFR2 and in the presence of JAK2 (Janus kinase 2) and tyrosine-protein kinase Src [62].

In addition to the main signaling pathways, other signaling events downstream of FGFRs have been described. Interestingly, SHB (SH2 domain-containing adaptor protein B) has been shown to bind phosphorylated Y766 in FGFR1 and might contribute to activation of the RAS/MAPK pathway via FRS2 $\alpha$ phosphorylation [63]. It is not well understood how binding of SHB and PLCY to the same phosphorylated tyrosine (Y766) in FGFR1 is regulated. The adaptor proteins, CRK and CRKL (CRK-like proto-oncogene), bind directly to phosphorylated Y463 in FGFR1 [64, 65]. FGFR activation and subsequent phosphorylation of FRS2 might lead to a release of CKS1 (cyclin-dependent kinase regulatory subunit 1) from FRS2. The release of CKS1 leads to ubiquitination and degradation of the cell cycle regulatory protein p27kip1 
(cyclin-dependent kinase inhibitor 1B). p27kip1 is a negative regulator of the $\mathrm{G} 1 / \mathrm{S}$ transition during cell cycle and therefore possibly mediates mitogenic signals from FGFR1 [66]. Activation of FGFRs can also lead to phosphorylation of focal adhesion kinase (FAK) [67].

Despite the fact that FGFRs usually activate only four main signaling pathways in addition to some signaling molecules, their activation influences a plethora of cellular functions in the human embryonic, fetal, and adult life.

\section{Noncanonical FGFR signaling}

Several atypical receptors and cell-surface proteins can interact with FGFRs/FGFs and might play a role in FGFR signaling. It has been shown that the transmembrane cysteine-rich FGF receptor (CFR) can bind FGF1, FGF2, FGF3, and FGF18 [68, 69, 70]. The role of CFR in FGFR signaling is not clear but it has been shown that binding of FGF18 to CFR enhanced FGF18-dependent cell proliferation. Integrins, receptors for extracellular matrix molecules, can also form signaling complexes with FGFR1 [71]. A complex of av $\beta 3$ integrin and FGFR1 seems to increase FGF2-induced ERK1/2 activation [72]. Moreover, receptors like the tyrosine kinase receptor, ephrin type-A receptor 4 (EphA4), and the G-protein coupled receptor, adenosine $A 2 A$, can also interact with FGFRs and influence activation and downstream signaling [73, 74].

It has also been demonstrated that FGFRs can be activated by noncanonical ligands such as cell adhesion molecules (CAMs) including L1, N-cadherin, integrins and neural cell adhesion molecules (NCAM) [75, 76]. N-cadherin is a transmembrane adhesion protein that has been shown to play a role in FGF-dependent and independent activation of FGFRs [77]. In cancer cells, N-cadherin has been shown to decrease ligand-induced internalization and lysosomal degradation of FGFR1, resulting in prolonged signaling [78]. Stimulation of FGFRs by NCAM has been shown to alter FGFR1 dynamics at the cell surface, leading to a changed cellular response compared to FGF2 stimulation [79, 80]. FGF2 induces dimerization and lysosomal degradation of FGFR1, while NCAM induces receptor recycling [79, 80]. NCAM-induced recycling of FGFR1 to the cell surface requires RAB11 and Src kinase and leads to sustained signaling [79]. NCAM can also form a complex with FGFR4 and N-cadherin [81].

\section{Negative regulation of FGFRs}

Many mechanisms exist to down-regulate FGFR signaling [87]. Tight regulation of signaling is necessary to maintain homeostasis in the body. The regulation of FGFR activity is not fully understood. It has been shown that certain molecules such as Sprouty $[82,83]$ and related protein SPRED (Sprouty related with EVH1 [Ena/VASP homology 1]), SEF (similar expression to Fgf), ubiquitin ligase CBL [11], phosphatases SHP2 [56], PTPRG (protein tyrosine phosphatase receptor type G) [84], and MKP3 (mitogen-activated protein kinase phosphatase 3) [85] act at different steps in the signaling pathways and may down-regulate FGFR signaling. However, the main mechanism that regulates the duration and strength of receptor signaling is endocytosis and subsequently its lysosomal degradation [87].

\section{Future perspective}

The FGF/FGFR signaling system is complex and has to work precisely in order to prevent diseases such as dwarfism and cancer. Therefore, a better understanding of the signaling, and the regulation of the FGF/FGFR signaling, is needed. Questions such as how the FGF/FGFR family can generate a plethora of biological effects in different receiver cells, using the same pathways, should be explored. Moreover, research on possible ways to manipulate the FGF/FGFR signaling system (such as inhibitors, etc.) should have the highest priority. Inappropriate signaling must be controlled to stop diseases [86].

\section{Abbreviations}

aFGF - acidic fibroblast growth factor, BAD - Bcl-2 antagonist of cell death, bFGF - basic fibroblast growth factor, CAM-cell adhesion molecule, CFR - cysteine-rich FGF receptor, Cks1 cyclin-dependent kinase regulatory subunit 1, CRKL-CRK-like proto-oncogene, DAG - diacylglycerol, EphA4 - ephrin type-A receptor 4, ER - endoplasmic reticulum, ERK - extracellular signal-regulated kinase, ETS - E26 transformation-specific, FAK - focal adhesion kinase, FGF - fibroblast growth factor, FGFR-fibroblastgrowth factor receptor, FRS2-FGFR substrate 2, GAB1 - GRB2-associated-binding protein1, GRB2 - growth factor receptor-bound 2, Hp - heparin, HS - heparan sulfate, HSPG - heparan sulfate proteoglycan, Ig - immunoglobulin, $\mathbf{I P}_{3}$ - inositol triphosphate, JAK - Janus kinase, JNK - c-Jun $\mathrm{N}$-terminal kinase, KLPH - Klotho-LPH related protein, MAPK - mitogen-activated protein kinase, MEK - mitogenactivated protein kinase kinase, MKP3 - MAPK phosphatase 3, mTOR - mammalian target of rapamycin, NCAM - neural cell adhesion molecules, p27kip1 - cyclin-dependent kinase inhibitor 1B, PDK1 - phosphoinositide-dependent kinase 1, PI3K - phosphatidylinositol-4,5-bisphosphate 3-kinase, $\mathbf{P I P}_{2}$ - Phosphatidylinositol-3,4-bisphosphate, $\mathbf{P I P}_{3}$ - phosphatidylinositol-3,4,5-trisphosphate, PKB - protein 
kinase B, PKC - protein kinase C, PLC $y$ - phospholipase C $\gamma$, PLSD-SD - platyspondylic lethal skeletal dysplasia, San Diego type, PTB - phosphotyrosine binding, PTPN - protein tyrosine phosphatase nonreceptor type, PTPR - protein tyrosine phosphatase receptor type, RSK - ribosomal s6 kinase, SEF - similar expression to Fgf, SH - Src homology, SHB - SH2 domain-containing adaptor protein B, SHP - Src homology region 2 domain-containing phosphatase, sos - son of sevenless homology, SPRED - sprouty related with EVH1 (Ena/VASP homology 1), SPRY - sprout, STAT - signal transducer and activator of transcription, TSC2 - tuberous sclerosis complex 2 .

\section{Authors' Contributions}

P.S.: writing the manuscript, literature review, designing the figures, final proofreading, and approval of the version for publication; E.M.H.: writing the manuscript, designing the figures, final proofreading, and approval of the version for publication; A.W.: research concept and design, literature review, final proofreading, and approval of the version for publication.

\section{Conflict of Interest}

The authors have no potential conflicts of interest to declare.

\section{References}

[1] Itoh N., Ornitz D.M.: Evolution of the Fgf and Fgfr gene families. Trends Genet., 2004; 20: 563-569

[2] Ogawa K., Kobayashi C., Hayashi T., Orii H., Watanabe K., Agata K.: Planarian fibroblast growth factor receptor homologs expressed in stem cells and cephalic ganglions. Dev. Growth Differ., 2002; 44: 191-204

[3] Itoh N., Ornitz D.M.: Fibroblast growth factors: From molecular evolution to roles in development, metabolism and disease. J. Biochem., 2011; 149: 121-130

[4] Itoh N., Ornitz D.M.: Functional evolutionary history of the mouse Fgf gene family. Dev. Dyn., 2008; 237: 18-27

[5] Mason I.: Initiation to end point: The multiple roles of fibroblast growth factors in neural development. Nat. Rev. Neurosci., 2007; 8: $583-596$

[6] Pourquié O.: Vertebrate segmentation: From cyclic gene networks to scoliosis. Cell, 2011; 145: 650-663

[7] Thisse B., Thisse C.: Functions and regulations of fibroblast growth factor signaling during embryonic development. Dev. Biol., 2005; 287: $390-402$
[8] Tulin S., Stathopoulos A.: Extending the family table: Insights from beyond vertebrates into the regulation of embryonic development by FGFs. Birth Defects Res C Embryo Today, 2010; 90: 214-227

[9] Dailey L., Ambrosetti D., Mansukhani A., Basilico C.: Mechanisms underlying differential responses to FGF signaling. Cytokine Growth Factor Rev., 2005; 16: 233-247

[10] Beenken A., Mohammadi M.: The FGF family: Biology, pathophysiology and therapy. Nat. Rev. Drug Discov., 2009; 8: 235-253

[11] Ornitz D.M., Itoh N.: The fibroblast growth factor signaling pathway. Wiley Interdiscip. Rev. Dev. Biol., 2015; 4: 215-266

[12] Ornitz D.M., Itoh N.: Fibroblast growth factors. Genome Biol., 2001; 2: 3005

[13] Powers C.J., McLeskey S.W., Wellstein A.: Fibroblast growth factors, their receptors and signaling. Endocr. Relat. Cancer, 2000; 7: $165-197$

[14] Blaber M., DiSalvo J., Thomas K.A.: X-ray crystal structure of human acidic fibroblast growth factor. Biochemistry, 1996; 35: 20862094

[15] Murzin A.G., Lesk A.M., Chothia C.: $\beta$-Trefoil fold. Patterns of structure and sequence in the Kunitz inhibitors interleukins-1 $\beta$ and $1 \alpha$ and fibroblast growth factors. J. Mol. Biol., 1992; 223: 531-543

[16] Gosavi S., Whitford P.C., Jennings P.A., Onuchic J.N.: Extracting function from a beta-trefoil folding motif. Proc. Natl. Acad. Sci. USA, 2008; 105: 10384-10389

[17] Laestander C., Engström W.: Role of fibroblast growth factors in elicitation of cell responses. Cell Prolif., 2014; 47: 3-11

[18] Nickel W., Seedorf M.: Unconventional mechanisms of protein transport to the cell surface of eukaryotic cells. Annu. Rev. Cell Dev. Biol., 2008; 24: 287-308

[19] Prudovsky I., Tarantini F., Landriscina M., Neivandt D., Soldi R., Kirov A., Small D., Kathir K.M., Rajalingam D., Kumar T.K.: Secretion without Golgi. J. Cell. Biochem., 2008; 103: 1327-1343

[20] Ornitz D.M.: FGFs, heparan sulfate and FGFRs: Complex interactions essential for development. Bioessays, 2000; 22: 108-112

[21] Makarenkova H.P., Hoffman M.P., Beenken A., Eliseenkova A.V., Meech R., Tsau C., Patel V.N., Lang R.A., Mohammadi M.: Differential interactions of FGFs with heparan sulfate control gradient formation and branching morphogenesis. Sci. Signal., 2009; 2: ra55

[22] Pellegrini L.: Role of heparan sulfate in fibroblast growth factor signalling: A structural view. Curr. Opin. Struct. Biol., 2001; 11: 629-634

[23] Zhang F., Zhang Z., Lin X., Beenken A., Eliseenkova A.V., Mohammadi M., Linhardt R.J.: Compositional analysis of heparin/ heparan sulfate interacting with fibroblast growth factorfibroblast growth factor receptor complexes. Biochemistry, 2009; 48: 83798386

[24] Itoh N.: Hormone-like (endocrine) FGFs: Their evolutionary history and roles in development, metabolism, and disease. Cell Tissue Res., 2010; 342: 1-11 
[25] Itoh N., Ohta H., Konishi M.: Endocrine FGFs: Evolution, physiology, pathophysiology, and pharmacotherapy. Front. Endocrinol., 2015; 6: 154

[26] Medici D., Razzaque M.S., Deluca S., Rector T.L., Hou B., Kang K., Goetz R., Mohammadi M., Kuro-o M., Olsen B.R., Lanske B.: FGF-23-Klotho signaling stimulates proliferation and prevents vitamin D-induced apoptosis. J. Cell Biol., 2008; 182: 459-465

[27] Goetz R., Dover K., Laezza F., Shtraizent N., Huang X., Tchetchik D., Eliseenkova A.V., Xu C.F., Neubert T.A., Ornitz D.M., Goldfarb M., Mohammadi M.: Crystal structure of a fibroblast growth factor homologous factor (FHF) defines a conserved surface on FHFs for binding and modulation of voltage-gated sodium channels. J. Biol. Chem., 2009; 284: 17883-17896

[28] Olsen S.K., Garbi M., Zampieri N., Eliseenkova A.V., Ornitz D.M., Goldfarb M., Mohammadi M.: Fibroblast growth factor (FGF) homologous factors share structural but not functional homology with FGFs. J. Biol. Chem., 2003; 278: 34226-34236

[29] Goldfarb M., Schoorlemmer J., Williams A., Diwakar S., Wang Q., Huang X., Giza J., Tchetchik D., Kelley K., Vega A., Matthews G., Rossi P., Ornitz D.M., D'Angelo E.: Fibroblast growth factor homologous factors control neuronal excitability through modulation of voltage-gated sodium channels. Neuron, 2007; 55: 449-463

[30] Lou J.Y., Laezza F., Gerber B.R., Xiao M., Yamada K.A., Hartmann H., Craig A.M., Nerbonne J.M., Ornitz D.M.: Fibroblast growth factor 14 is an intracellular modulator of voltage-gated sodium channels. J. Physiol., 2005; 569: 179-193

[31] Schoorlemmer J., Goldfarb M.: Fibroblast growth factor homologous factors are intracellular signaling proteins. Curr. Biol., 2001; 11: 793-797

[32] Bruns A.F., van Bergeijk J., Lorbeer C., Nölle A., Jungnickel J., Grothe C., Claus P.: Fibroblast growth factor-2 regulates the stability of nuclear bodies. Proc. Natl. Acad. Sci. USA, 2009; 106: 12747-12752

[33] Kiefer P., Acland P., Pappin D., Peters G., Dickson C.: Competition between nuclear localization and secretory signals determines the subcellular fate of a single CUG-initiated form of FGF3. EMBO J., 1994; 13: 4126-4136

[34] Kostas M., Lampart A., Bober J., Wiedlocha A., Tomala J., Krowarsch D., Otlewski J., Zakrzewska M.: Translocation of exogenous FGF1 and FGF2 protects the cell against apoptosis independently of receptor activation. J. Mol. Biol., 2018; 430: 4087-4101

[35] Sørensen V., Nilsen T., Wiedłocha A.: Functional diversity of FGF2 isoforms by intracellular sorting. Bioessays, 2006; 28: 504-514

[36] Sørensen V., Zhen Y., Zakrzewska M., Haugsten E.M., Wälchli S., Nilsen T., Olsnes S., Wiedlocha A.: Phosphorylation of fibroblast growth factor (FGF) receptor 1 at Ser777 by p38 mitogen-activated protein kinase regulates translocation of exogenous FGF1 to the cytosol and nucleus. Mol. Cell. Biol., 2008; 28: 4129-4141

[37] Sluzalska K.D., Slawski J., Sochacka M., Lampart A., Otlewski J., Zakrzewska M.: Intracellular partners of fibroblast growth factors 1 and 2 - implications for functions. Cytokine Growth Factor Rev., 2021; 57: 93-111
[38] Eswarakumar V.P., Lax I., Schlessinger J.: Cellular signaling by fibroblast growth factor receptors. Cytokine Growth Factor Rev., 2005; 16: 139-149

[39] Lemmon M.A., Schlessinger J.: Cell signaling by receptor tyrosine kinases. Cell, 2010; 141: 1117-1134

[40] Sarrazin S., Lamanna W.C., Esko J.D.: Heparan sulfate proteoglycans. Cold Spring Harb. Perspect. Biol., 2011; 3: a004952

[41] Harmer N.J.: Insights into the role of heparan sulphate in fibroblast growth factor signalling. Biochem. Soc. Trans., 2006; 34: 442-445

[42] Johnson D.E., Williams L.T.: Structural and functional diversity in the FGF receptor multigene family. Adv. Cancer Res., 1993; 60: $1-41$

[43] Scotet E., Houssaint E.: The choice between alternative IIIb and Illc exons of the FGFR-3 gene is not strictly tissue-specific. Biochim. Biophys. Acta, 1995; 1264: 238-242

[44] Pellegrini L., Burke D.F., von Delft F., Mulloy B., Blundell T.L.: Crystal structure of fibroblast growth factor receptor ectodomain bound to ligand and heparin. Nature, 2000; 407: 1029-1034

[45] Schlessinger J., Plotnikov A.N., Ibrahimi O.A., Eliseenkova A.V., Yeh B.K., Yayon A., Linhardt R.J., Mohammadi M.: Crystal structure of a ternary FGF-FGFR-heparin complex reveals a dual role for heparin in FGFR binding and dimerization. Mol. Cell, 2000; 6: 743-750

[46] Mohammadi M., Olsen S.K., Goetz R.: A protein canyon in the FGF-FGF receptor dimer selects from an à la carte menu of heparan sulfate motifs. Curr. Opin. Struct. Biol., 2005; 15: 506-516

[47] Paul M.D., Hristova K.: The RTK interactome: Overview and perspective on RTK heterointeractions. Chem. Rev., 2019; 119: 5881-5921

[48] Sarabipour S., Hristova K.: Mechanism of FGF receptor dimerization and activation. Nat. Commun., 2016; 7: 10262

[49] Brown A., Robinson C.J., Gallagher J.T., Blundell T.L.: Cooperative heparin-mediated oligomerization of fibroblast growth factor-1 (FGF1) precedes recruitment of FGFR2 to ternary complexes. Biophys. J., 2013; 104: 1720-1730

[50] Pomin V.H.: Paradigms in the structural biology of the mitogenic ternary complex FGF:FGFR:heparin. Biochimie, 2016; 127: 214226

[51] Patel V.N., Likar K.M., Zisman-Rozen S., Cowherd S.N., Lassiter K.S., Sher I., Yates E.A., Turnbull J.E., Ron D., Hoffman M.P.: Specific heparan sulfate structures modulate FGF10-mediated submandibular gland epithelial morphogenesis and differentiation. J. Biol. Chem., 2008; 283: 9308-9317

[52] Goetz R., Mohammadi M.: Exploring mechanisms of FGF signalling through the lens of structural biology. Nat. Rev. Mol. Cell Biol., 2013; 14: 166-180

[53] Mohammadi M., Dikic I., Sorokin A., Burgess W.H., Jaye M., Schlessinger J.: Identification of six novel autophosphorylation sites on fibroblast growth factor receptor 1 and elucidation of their importance in receptor activation and signal transduction. Mol. Cell. Biol., 1996; 16: 977-989 
[54] Mohammadi M., Honegger A.M., Rotin D., Fischer R., Bellot F., Li W., Dionne C.A., Jaye M., Rubinstein M., Schlessinger J.: A tyrosine-phosphorylated carboxy-terminal peptide of the fibroblast growth factor receptor $(\mathrm{Flg})$ is a binding site for the $\mathrm{SH} 2$ domain of phospholipase C-gamma 1. Mol. Cell. Biol., 1991; 11: 5068-5078

[55] Furdui C.M., Lew E.D., Schlessinger J., Anderson K.S.: Autophosphorylation of FGFR1 kinase is mediated by a sequential and precisely ordered reaction. Mol. Cell, 2006; 21: 711-717

[56] Hadari Y.R., Kouhara H., Lax I., Schlessinger J.: Binding of Shp2 tyrosine phosphatase to FRS2 is essential for fibroblast growth factor-induced PC12 cell differentiation. Mol. Cell. Biol., 1998; 18: 3966-3973

[57] Kouhara H., Hadari Y.R., Spivak-Kroizman T., Schilling J., BarSagi D., Lax I., Schlessinger J.: A lipid-anchored Grb2-binding protein that links FGF-receptor activation to the Ras/MAPK signaling pathway. Cell, 1997; 89: 693-702

[58] Lanner F., Rossant J.: The role of FGF/Erk signaling in pluripotent cells. Development, 2010; 137: 3351-3360

[59] Lamothe B., Yamada M., Schaeper U., Birchmeier W., Lax I., Schlessinger J.: The docking protein Gab1 is an essential component of an indirect mechanism for fibroblast growth factor stimulation of the phosphatidylinositol 3-kinase/Akt antiapoptotic pathway. Mol. Cell. Biol., 2004; 24: 5657-5666

[60] Manning B.D., Cantley L.C.: AKT/PKB signaling: Navigating downstream. Cell, 2007; 129: 1261-1274

[61] Hung C.M., Garcia-Haro L., Sparks C.A., Guertin D.A.: mTORdependent cell survival mechanisms. Cold Spring Harb. Perspect. Biol., 2012; 4: a008771

[62] Dudka A.A., Sweet S.M., Heath J.K.: Signal transducers and activators of transcription-3 binding to the fibroblast growth factor receptor is activated by receptor amplification. Cancer Res., 2010; 70: $3391-3401$

[63] Cross M.J., Lu L., Magnusson P., Nyqvist D., Holmqvist K., Welsh M., Claesson-Welsh L.: The Shb adaptor protein binds to tyrosine 766 in the FGFR-1 and regulates the Ras/MEK/MAPK pathway via FRS2 phosphorylation in endothelial cells. Mol. Biol. Cell, 2002; 13: $2881-2893$

[64] Larsson H., Klint P., Landgren E., Claesson-Welsh L.: Fibroblast growth factor receptor-1-mediated endothelial cell proliferation is dependent on the Src homology (SH) $2 / \mathrm{SH} 3$ domain-containing adaptor protein Crk. J. Biol. Chem., 1999; 274: 25726-25734

[65] Seo J.H., Suenaga A., Hatakeyama M., Taiji M., Imamoto A.: Structural and functional basis of a role for CRKL in a fibroblast growth factor 8-induced feed-forward loop. Mol. Cell. Biol., 2009; 29: $3076-3087$

[66] Zhang Y., Lin Y., Bowles C., Wang F.: Direct cell cycle regulation by the fibroblast growth factor receptor (FGFR) kinase through phosphorylation-dependent release of Cks1 from FGFR substrate 2. J. Biol. Chem., 2004; 279: 55348-55354

[67] Hatai M., Hashi H., Mogi A., Soga H., Yokota J., Yaoi Y.: Stimulation of tyrosine- and serine-phosphorylation of focal adhesion kinase in mouse 3T3 cells by fibronectin and fibroblast growth factor. FEBS Lett., 1994; 350: 113-116

[68] Burrus L.W., Zuber M.E., Lueddecke B.A., Olwin B.B.: Identification of a cysteine-rich receptor for fibroblast growth factors. Mol. Cell. Biol., 1992; 12: 5600-5609

[69] Köhl R., Antoine M., Olwin B.B., Dickson C., Kiefer P.: Cysteinerich fibroblast growth factor receptor alters secretion and intracellular routing of fibroblast growth factor 3. J. Biol. Chem., 2000; 275: 15741-15748

[70] Miyaoka Y., Tanaka M., Imamura T., Takada S., Miyajima A.: A novel regulatory mechanism for Fgf18 signaling involving cysteine-rich FGF receptor (Cfr) and delta-like protein (DIk). Development, 2010; 137: 159-167

[71] Klein S., Bikfalvi A., Birkenmeier T.M., Giancotti F.G., Rifkin D.B.: Integrin regulation by endogenous expression of $18-\mathrm{kDa}$ fibroblast growth factor-2. J. Biol. Chem., 1996; 271: 22583-22590

[72] Tanghetti E., Ria R., Dell'Era P., Urbinati C., Rusnati M., Ennas M.G., Presta M.: Biological activity of substrate-bound basic fibroblast growth factor (FGF2): Recruitment of FGF receptor-1 in endothelial cell adhesion contacts. Oncogene, 2002; 21: 3889-3897

[73] Flajolet M., Wang Z., Futter M., Shen W., Nuangchamnong N., Bendor J., Wallach I., Nairn A.C., Surmeier D.J., Greengard P.: FGF acts as a co-transmitter through adenosine $\mathrm{A}(2 \mathrm{~A})$ receptor to regulate synaptic plasticity. Nat. Neurosci., 2008; 11: 1402-1409

[74] Yokote H., Fujita K., Jing X., Sawada T., Liang S., Yao L., Yan X., Zhang Y., Schlessinger J., Sakaguchi K.: Trans-activation of EphA4 and FGF receptors mediated by direct interactions between their cytoplasmic domains. Proc. Natl. Acad. Sci. USA 2005; 102: 18866-18871

[75] Kiselyov V.V., Skladchikova G., Hinsby A.M., Jensen P.H., Kulahin N., Soroka V., Pedersen N., Tsetlin V., Poulsen F.M., Berezin V., Bock E.: Structural basis for a direct interaction between FGFR1 and NCAM and evidence for a regulatory role of ATP. Structure, 2003; 11: 691-701

[76] Kulahin N., Li S., Hinsby A., Kiselyov V., Berezin V., Bock E.: Fibronectin type III (FN3) modules of the neuronal cell adhesion molecule L1 interact directly with the fibroblast growth factor (FGF) receptor. Mol. Cell. Neurosci., 2008; 37: 528-536

[77] Murakami M., Elfenbein A., Simons M.: Non-canonical fibroblast growth factor signalling in angiogenesis. Cardiovasc. Res., 2008; 78: 223-231

[78] Suyama K., Shapiro I., Guttman M., Hazan R.B.: A signaling pathway leading to metastasis is controlled by $\mathrm{N}$-cadherin and the FGF receptor. Cancer Cell, 2002; 2: 301-314

[79] Francavilla C., Cattaneo P., Berezin V., Bock E., Ami D., de Marco A., Christofori G., Cavallaro U.: The binding of NCAM to FGFR1 induces a specific cellular response mediated by receptor trafficking. J. Cell Biol., 2009; 187: 1101-1116

[80] Zamai M., Trullo A., Giordano M., Corti V., Cuesta E.A., Francavilla C., Cavallaro U., Caiolfa V.R.: Number and brightness analysis reveals that NCAM and FGF2 elicit different assembly and dynamics of FGFR1 in live cells. J. Cell Sci., 2019; 132: jcs220624 
[81] Cavallaro U., Niedermeyer J., Fuxa M., Christofori G.: N-CAM modulates tumour-cell adhesion to matrix by inducing FGF-receptor signalling. Nat. Cell Biol., 2001; 3: 650-657

[82] Dikic I., Giordano S.: Negative receptor signalling. Curr. Opin. Cell Biol., 2003; 15: 128-135

[83] Yim D.G., Ghosh S., Guy G.R., Virshup D.M.: Casein kinase 1 regulates Sprouty2 in FGF-ERK signaling. Oncogene, 2015; 34: 474-484

[84] Kostas M., Haugsten E.M., Zhen Y., Sørensen V., Szybowska P., Fiorito E., Lorenz S., Jones N., de Souza G.A., Wiedlocha A.,Wesche J.: Protein tyrosine phosphatase receptor type G (PTPRG) controls fibroblast growth factor receptor (FGFR) 1 activity and influences sensitivity to FGFR kinase inhibitors. Mol. Cell. Proteomics, 2018; 17: 850-870

[85] Camps M., Nichols A., Gillieron C., Antonsson B., Muda M., Chabert C., Boschert U., Arkinstall S.: Catalytic activation of the phosphatase MKP-3 by ERK2 mitogen-activated protein kinase. Science, 1998; 280: 1262-1265

[86] Turner N., Grose R.: Fibroblast growth factor signalling: From development to cancer. Nat. Rev. Cancer, 2010; 10: 116-129

[87] Szybowska P., Kostas M., Wesche J., Haugsten E., M., Wiedlocha A.: Negative regulation of FGFR (Fibroblast Growth Factor Receptor) signaling. Cells, 2021; 10;1342 